\title{
Clinical Results and Failure Mechanisms of a Nonmodular Constrained Knee Without Stem Extensions
}

\author{
Denis Nam, MD • Ben-Paul N. Umunna, BA • Michael B. Cross, MD • Keith R. Reinhardt, MD • Shivi Duggal, BA • \\ Charles N. Cornell, MD
}

Received: 5 December 2011/Accepted: 10 April 2012/Published online: 12 May 2012

C Hospital for Special Surgery 2012

\begin{abstract}
Introduction: In the setting of persistent knee instability despite appropriate ligament balancing for primary total knee arthroplasty, most surgeons advocate the use of an implant with increased articular constraint. These implants are commonly supplemented with stem extensions to improve stress transfer and decrease the risk of aseptic loosening. However, disadvantages exist with the use of stem extensions, including increased cost, intramedullary invasion, and diaphyseal pain. The objectives of this study were to (1) compare the clinical results as assessed by the Knee Society, Hospital for Special Surgery, and SF-12 scores, (2) determine the incidence of failure as defined by the need for a revision procedure, and (3) to analyze the causes or modes of failure of a nonmodular constrained condylar knee without the use of stem extensions versus a conventional, posterior-stabilized design. Materials and Methods: From 2002 to 2007, 190 TKAs were implanted using a primary, nonmodular constrained (NMC) prosthesis without stem extensions. During the same time period, clinical data were available for 140 TKAs implanted using a standard, posterior-stabilized (PS) design. Preoperative demographic data was reviewed, in addition to the rate and reason for revision in each cohort. Clinical data included HSS, Knee Society, and SF-12 scores at the latest follow-up, and the results of the NMC and PS cohorts were statistically compared using a Student's twotailed $t$ test. Results: The mean age of patients in the NMC cohort was $72.3 \pm 10.2$ years, and the mean length of follow-up
\end{abstract}

Level of Evidence: Therapeutic Study: Level III. See Levels of Evidence for a complete description.

D. Nam MD $(\bowtie) \cdot$ B.-P. N. Umunna BA • M. B. Cross MD •

K. R. Reinhardt MD $\cdot$ S. Duggal BA $\cdot$ C. N. Cornell MD

Hospital for Special Surgery,

535 East 70th Street, New York, NY 10021, USA

e-mail: namd@hss.edu

D. Nam MD • M. B. Cross MD • K. R. Reinhardt MD •

C. N. Cornell MD

Weill Cornell Medical College, New York, NY 10065, USA was $7.3 \pm 2.1$ years. The mean age of the PS cohort was $67.1 \pm$ 8.7 years, with a mean follow-up of $6.1 \pm 2.2$ years. No statistically significant differences in the HSS, Knee Society, or SF12 scores were appreciated between the two cohorts. The revision rate in the NMC cohort was $4.2 \%$ compared to $4.3 \%$ for the PS cohort. The most common cause of failure in the NMC cohort was femoral component loosening, all of which occurred when Palacos cement was used for fixation. NMC components (55.6\%) implanted with Palacos cement failed due to femoral component loosening. In contrast, all PS components requiring revision were revised for persistent instability. Discussion: At mid-term follow-up, NMC prostheses without stem extensions have excellent clinical results and are a viable option for patients with ligamentous instability. The use of Palacos cement in this scenario was associated with a high rate of femoral component loosening, possibly due to the decreased intrusion depth of Palacos when compared to Simplex cement.

Keywords nonmodular. constrained total condylar knee. component loosening $\cdot$ Palacos cement

\section{Introduction}

Achieving knee joint stability in total knee arthroplasty (TKA) is crucial for both postoperative function and implant longevity. In a retrospective review of 440 patients who required revision surgery within 5 years of their index arthroplasty, $27 \%$ were revised due to instability [6]. In the setting of a mild to moderate preoperative deformity, ligament balance in both flexion and extension can often be achieved via appropriate soft tissue balancing and subperiosteal releases, and thus, a posterior cruciate-retaining or a posterior cruciate-substituting knee can safely be implanted. However, in patients with persistent instability, whether secondary to severe preoperative deformity, bone loss, or poor tissue quality, an implant with increased articular constraint is often required [17].

The goal of increased articular constraint is to limit varus-valgus and torsional moments and thus provide 
increased stability to the knee. This is typically achieved via an implant with a tibial post that fits intimately between the femoral condyles and anterior to a nonlinked cam in the femoral component [1]. This increased constraint allows fewer degrees of motion versus a standard, posterior-stabilized implant, the extent of which varies among different implant designs. However, the use of increased articular constraint raises concerns of early implant loosening due to increased stress transferred to the bone-implant interface. Thus, most surgeons advocate the use of stem extensions with constrained components in order to distribute load from the bone-implant interface to the diaphyseal portions of the tibia and femur [2]. Constrained condylar knee (CCK) implants with stem extensions have shown good to excellent results in several studies in both the complex primary and revision TKA setting [5, 7, 10]. Easley et al. [5] reviewed the results of 28 primary CCK knees with noncemented stem extensions at a mean follow-up of 7.8 years and noted a mean improvement in Knee Society score from 27.4 preoperatively to 95.2 at latest follow-up, with no incidence of radiographic loosening or prosthetic failure.

However, disadvantages exist with the use of stem extensions, including increased cost, intramedullary invasion and possible embolization, difficulty of removal and additional bone loss in the revision setting, and a reported incidence of $15-20 \%$ of leg and thigh pain near the tips of the stems [3, 4, $7,17]$. Therefore, the use of constrained condylar knee implants without stem extensions has been implemented, and clinically, they have shown reliable short- to mid-term results [1, 2, 13, 17]. Anderson et al. [2] reviewed 192 constrained condylar knee arthroplasties without the use of diaphyseal stem extensions at a mean follow-up of 47 months and noted an increase in Knee Society Score from 36 to 89 points postoperatively, with a failure rate of $2.5 \%$. However, the results of that study were compared only to historical controls. The objectives of this study were to (1) compare the clinical results as assessed by the Knee Society, Hospital for Special Surgery, and SF-12 scores, (2) determine the incidence of failure as defined by the need for a revision procedure, and (3) analyze the causes or modes of failure of a nonmodular constrained condylar knee without the use of stem extensions versus a conventional, posterior-stabilized design.

\section{Materials and Methods}

From 2002 to 2007, 181 consecutive patients (65 male, 116 female: $36 \%$ male) undergoing primary total knee arthroplasty were enrolled in an Institutional Review Board-approved database of a single surgeon (CNC). Nine patients underwent bilateral TKAs, for a total of 190 knees (91 right and 99 left). All patients received a primary nonmodular constrained (NMC) prosthesis without stem extensions (Fig. 1) (Exactech, Inc., Gainesville, FL, USA). This type of constrained condylar implant was used to provide inherent knee stability in older patients with severe deformity and compromised collateral ligaments (Fig. 2). These patients typically had a more severe preoperative varus/valgus deformity (although no specific alignment cutoff was instituted), were older in age, and were more difficult to achieve intraoperative ligament balancing versus patients who received a conventional, posterior-stabilized design. The design of the nonmodular implant implements a femoral box that is $2 \mathrm{~mm}$ deeper than the box of a standard, posterior-stabilized design. A higher, more squared, tibial post is then implanted, which increases varus/valgus and anterior/posterior stability. The specific implant used in this study is called "nonmodular" as the femoral component does not allow for the use of stem extensions or metal augments. The tibial component has a standard $5-\mathrm{cm}$ stem, with modularity in the thickness of the polyethylene, and the post is reinforced by a screw to increase its resistance to bending [17].

During the same time period, clinical data on 133 patients (44 male, 89 female: $33 \%$ male) undergoing primary total knee arthroplasty who received a standard, posterior-stabilized (PS) TKA by the same surgeon were available for review (Fig. 3). Seven patients underwent bilateral TKAs, for a total of 140 knees (76 right and 64 left). Therefore, a cohort of 190 NMC knees and 140 PS knees were available for clinical review and comparison.

Preoperative demographic data collected included the patient's age, body mass index, and primary diagnosis. Standing anteroposterior (AP) knee radiographs were obtained for each patient, from which the lower extremity tibiofemoral anatomic alignment was measured (in degrees). The mean age of patients who received NMC prosthesis without stem extensions was $72.3 \pm 10.2$ years at the time of surgery, with a mean body mass index (BMI) of $30.9 \pm$ $7.1 \mathrm{~kg} / \mathrm{m}^{2}$. One hundred eighty patients had a primary

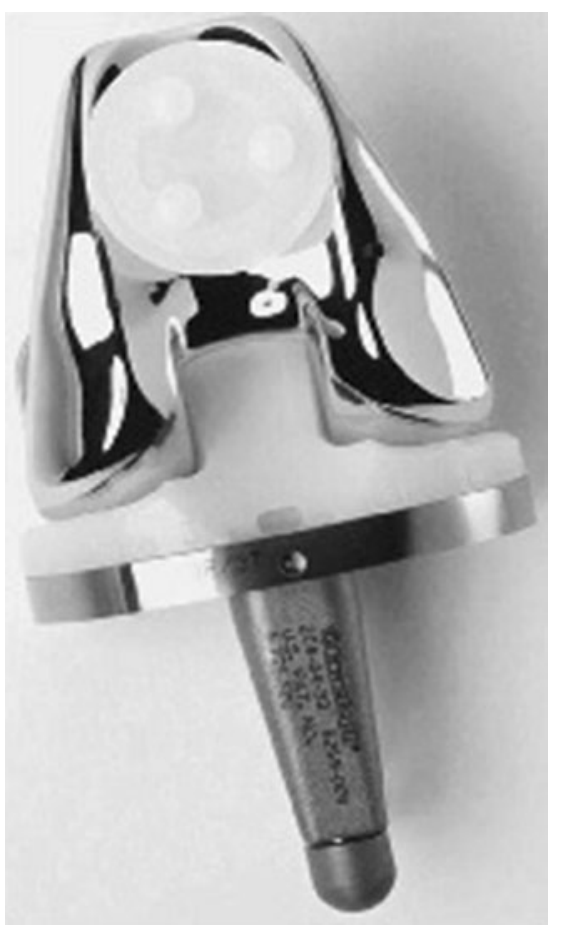

Fig. 1. Anterior view of the nonmodular constrained prosthesis (Exactech, Inc., Gainesville, FL, USA) (Reprinted with permission from Anderson JA, Baldini A, MacDonald JH, Pellicci PM, Sculco TP. Primary constrained condylar knee arthroplasty without stem extensions for the valgus knee. Clin Orthop Relat Res. 2006;442:199-203 [1] 


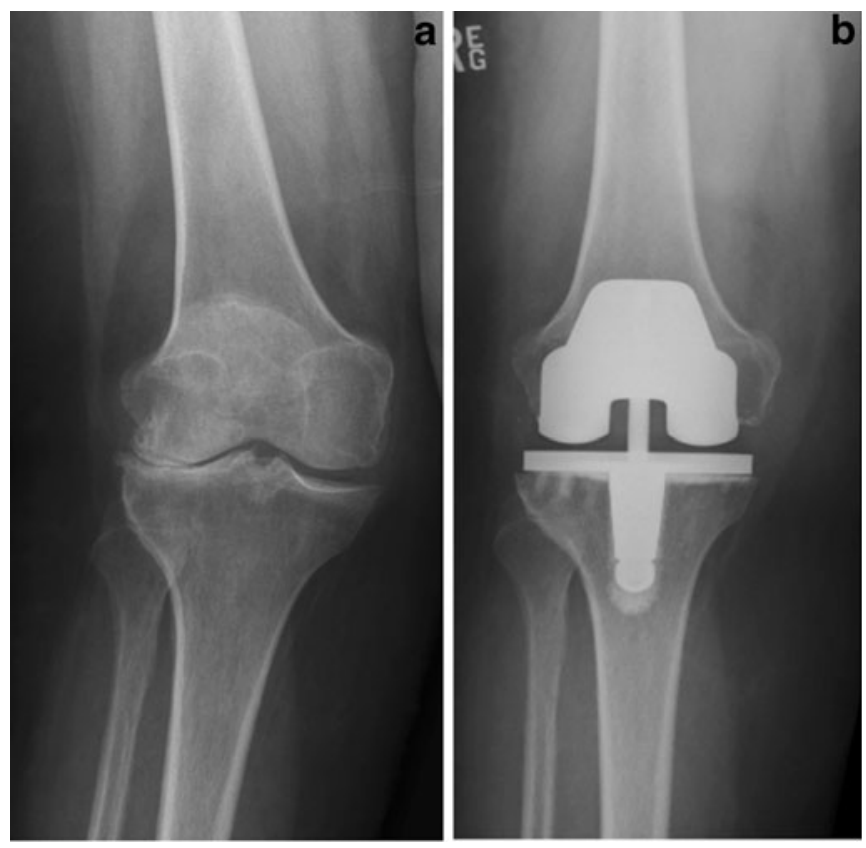

Fig. 2. a Preoperative AP radiograph of a severe valgus knee in a 72year-old woman. b Postoperative AP radiograph 5 years after implantation of NMC prosthesis without stem extensions

diagnosis of osteoarthritis, six post-traumatic arthritis after a tibial plateau fracture, three rheumatoid arthritis, and one gonococcal arthritis. The mean preoperative alignment for patients with a varus deformity was $10.2^{\circ} \pm 5.4^{\circ}$, and for patients with a valgus deformity was $15.5^{\circ} \pm 5.1^{\circ}$. The mean length of follow-up for the NMC cohort of patients was $7.3 \pm 2.1$ years (minimum, 3.8 years; Table 1 ).

The mean age of patients who received a standard, PS prosthesis was $67.1 \pm 8.7$ years, which was a significantly different compared to the NMC cohort $(p=0.04)$. The mean BMI of these patients was $31.3 \pm 5.7 \mathrm{~kg} / \mathrm{m}^{2}$, which was not significantly different than the NMC cohort $(p=0.74)$. One hundred thirty-five patients had a primary diagnosis of

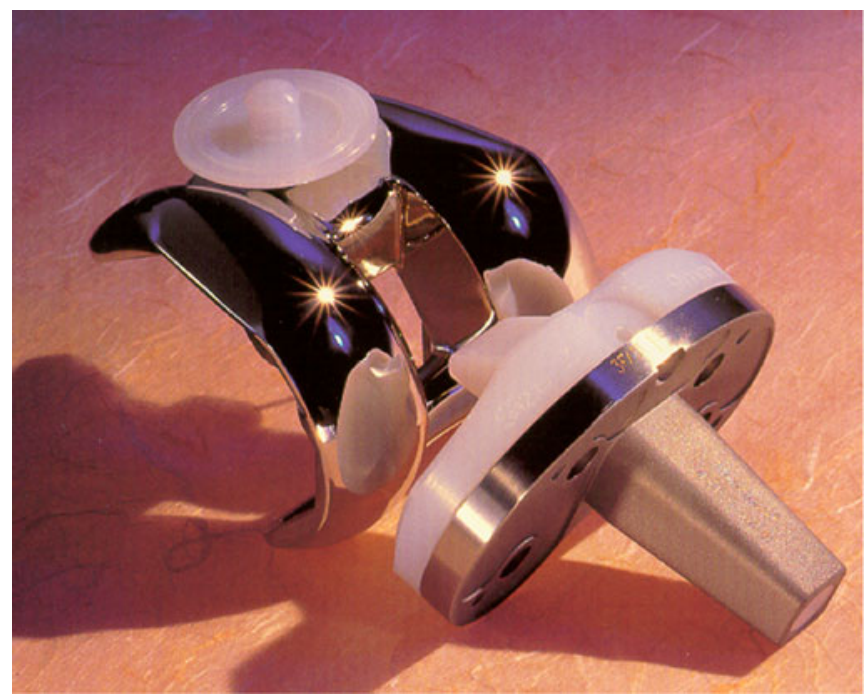

Fig. 3. Image of the standard, posterior-stabilized knee used in this study (Exactech, Inc., Gainesville, FL, USA)
Table 1 Table comparing the preoperative demographic variables and radiographic alignments of the NMC and PS cohorts

\begin{tabular}{lll}
\hline & NMC $(N=190)$ & PS $(N=140)$ \\
\hline Mean age (years) & $72.3 \pm 10.2$ & $67.1 \pm 8.7$ \\
Mean BMI $\left(\mathrm{kg} / \mathrm{m}^{2}\right)$ & $30.9 \pm 7.1$ & $31.3 \pm 5.7$ \\
Preoperative diagnosis & 180 & 135 \\
Osteoarthritis & 6 & 4 \\
Post-traumatic & 3 & 0 \\
Rheumatoid arthritis & 1 & 1 \\
Gonococcal arthritis & 0 & 0 \\
Prior high tibial osteotomy & $10.2 \pm 5.4$ & $7.1 \pm 4.5$ \\
Preoperative radiographic alignment $\left(^{\circ}\right)$ & $6.1 \pm 3.6$ \\
Varus $(p=0.03)$ & $15.5 \pm 5.1$ & $6.1 \pm 2.2$ \\
Valgus $(p=0.01)$ & $7.3 \pm 2.1$ & \\
Mean Follow-up & &
\end{tabular}

osteoarthritis, four had post-traumatic arthritis following a tibial plateau fracture, and one had a history of a prior high tibial osteotomy for varus malalignment. The mean preoperative alignment for patients with a varus deformity was $7.1^{\circ} \pm 4.5^{\circ}$, and for patients with a valgus deformity was $6.1^{\circ} \pm 3.6^{\circ}$. The differences in preoperative alignment between the PS and NMC groups were statistically significant for both the preoperative varus $(p=0.03)$ and valgus $(p=0.01)$ patients. The mean length of follow-up for the PS cohort of patients was $6.1 \pm 2.2$ years (minimum 3.6 years).

All procedures were performed using a straight skin incision and midvastus surgical approach by the senior author $(\mathrm{CNC})$. An extramedullary tibial cutting guide was used to create a resection perpendicular to the anatomic axis of the tibia, while an intramedullary alignment guide was used to perform the anterior rough cut and distal femoral resection. For knees with a preoperative varus alignment, the distal cut was made in $5^{\circ}$ of valgus relative to the anatomic axis of the femur, while in knees with a preoperative valgus alignment, the distal cut was made in $2^{\circ}$ of valgus relative to the anatomic axis of the femur. In all cases, rotation of the femoral component was set relative to Whiteside's line [19]. In each case, the femoral, tibial, and patella components were implanted using cement, and the patella was resurfaced using an all-polyethylene, 3-pegged button. The type of implant (NMC vs. PS), and the type of cement (Simplex, Stryker Inc., Mahwah, NJ, USA vs. Palacos, Zimmer Inc., Warsaw, IN, USA) was recorded. During cementation of the femoral component, cement was directly applied to the undersurface of the posterior femoral condyles and surrounding the box of the implant, with additional cement applied directly to the bony surfaces corresponding to the anterior flange and distal condyles. During cementation of the tibial component, cement was applied directly to the undersurface of the tibial tray, with additional cement placed on the proximal tibia. Of the patients who received NMC prostheses, nine were implanted using Palacos cement, while 181 were implanted using Simplex. Of the patients who received standard, PS prostheses, 21 were implanted using Palacos and 119 using Simplex.

At each patient's most recent follow-up visit, standing anteroposterior, lateral, and Merchant view knee radiographs 


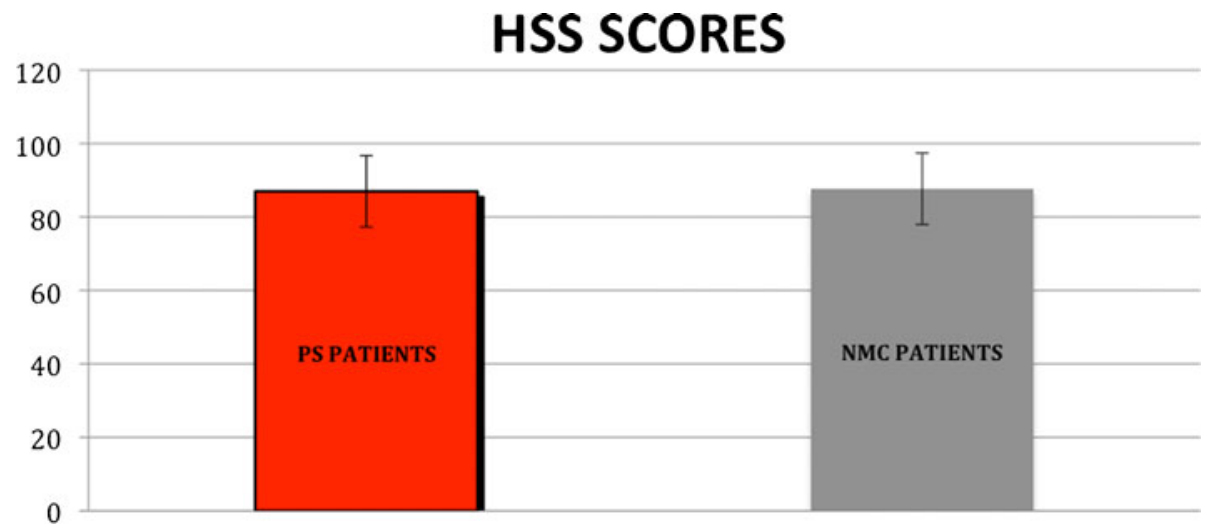

Fig. 4. Graph depicting the Hospital for Special Surgery Knee scores of both the PS cohort and NMC cohort at most recent follow-up. No statistically significant difference was appreciated between the two groups $(p=0.19)$

were obtained [12]. Postoperative tibiofemoral alignment was recorded. In addition, patients were clinically examined and questioned to obtain Knee Society (KSS) [8], Hospital for Special Surgery (HSS) [20], and mental and physical components of the SF-12 score. If a patient required a revision surgery, the revision diagnosis was recorded. Statistical comparisons of the pre- and postoperative clinical data between the two cohorts were performed using a Student's two-tailed $t$ test. Statistical significance was set at $p<0.05$.

\section{Results}

The mean postoperative radiographic alignment in the coronal plane was $4.2^{\circ} \pm 2.0^{\circ}$ of anatomic valgus for the NMC cohort and $3.5^{\circ} \pm 2.8^{\circ}$ of anatomic valgus for the PS cohort, a difference that was not statistically significant $(p=0.07)$.

There was no difference in clinical outcome as measured by the KSS, HSS, or the mental and physical components of the SF-12 score at the latest follow-up when the two designs were compared. At the most recent follow-up, the mean HSS Score was $87.7 \pm 9.7$ in the NMC group and $87.0 \pm 13.0$ in the PS group $(p=0.19)$, and the mean KSS Score was $87.7 \pm 14.5$ in the NMC group and $80.4 \pm 19.1$ in the PS group $(p=0.07)$ (Figs. 4 and 5). In addition, the mental component of the SF-
12 score was $51.3 \pm 9.9$ in the NMC group and $51.9 \pm 8.6$ in the PS group ( $p=0.93)$, and the physical component of the SF-12 score was $41.4 \pm 10.5$ in the NMC group and $43.8 \pm$ 10.6 in the PS group ( $p=0.11)$ (Fig. 6).

There was no difference in the incidence of failure (defined by the need for a revision surgery) when the two designs were compared, although the indications for revision between the NMC and PS cohorts demonstrated substantial differences. Eight out of 190 patients $(4.2 \%)$ who received a primary NMC implant without stem extensions required a revision surgery during the study period. Five patients were revised for femoral component loosening, one for infection, one for stiffness, and one for knee instability. Of note, all five patients revised for femoral component loosening had prostheses implanted using Palacos cement, and thus, five out of nine patients $(55.6 \%)$ who received NMC prostheses with Palacos cement were revised specifically for femoral component loosening. Six out of 140 patients $(4.3 \%)$ who received a standard, PS implant required a revision surgery during the study period. All six patients were revised due to persistent knee instability. There was no statistically significant difference in the BMI's of patients with NMC implants that required revision, or patients with standard, PS implants that required revision $\left(31.2 \pm 6.0 \mathrm{~kg} / \mathrm{m}^{2}\right.$ versus $28.8 \pm 4.3 \mathrm{~kg} / \mathrm{m}^{2}$, $p=0.48$ ).

KSS SCORES

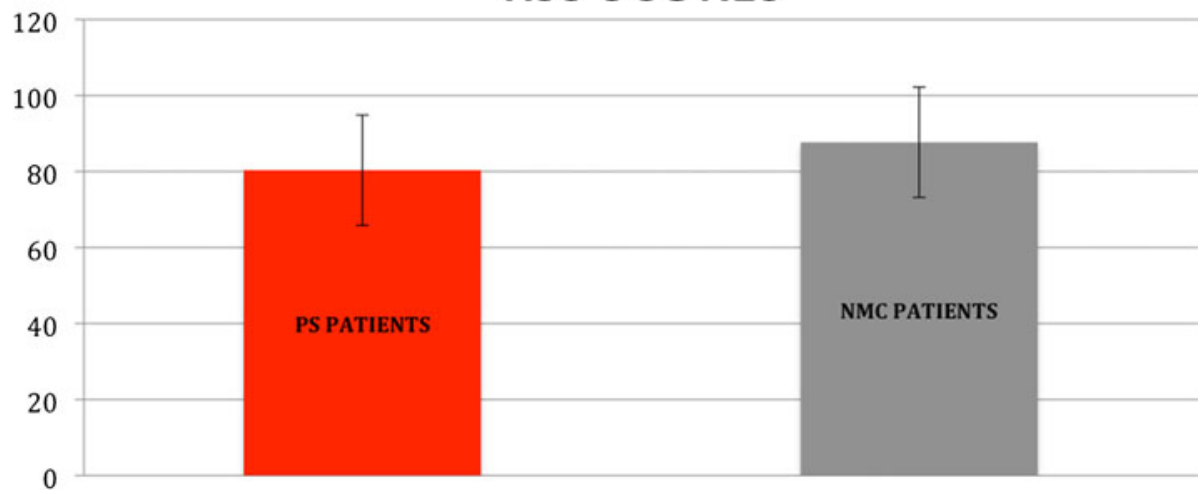

Fig. 5. Graph depicting the Knee Society scores of both the PS cohort and NMC cohort at most recent follow-up. No statistically significant difference was appreciated between the two groups $(p=0.07)$ 


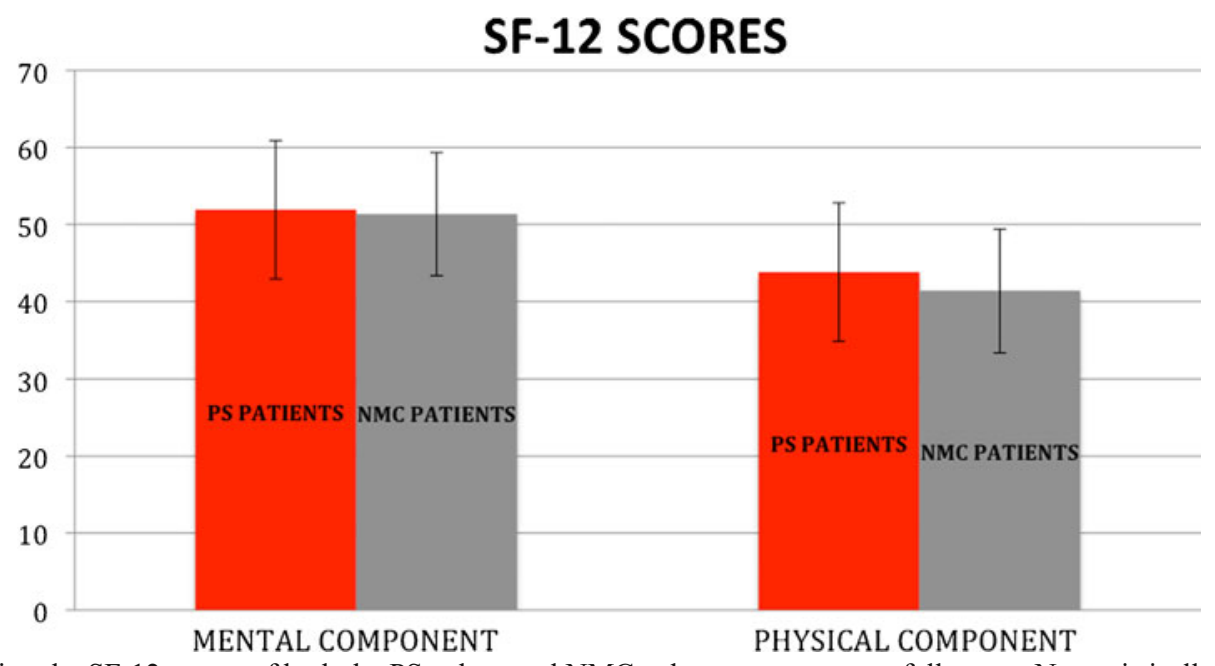

Fig. 6. Graph depicting the SF-12 scores of both the PS cohort and NMC cohort at most recent follow-up. No statistically significant difference was appreciated between the two groups for either the mental component $(p=0.93)$ or physical component $(p=0.11)$

\section{Discussion}

The objective of this study was to compare the clinical results at mid-term follow-up of a nonmodular constrained condylar knee without the use of stem extensions, to a conventional, posterior-stabilized design. In addition, the specific modes of failure and reasons for revision within each cohort were analyzed. This retrospective review demonstrates that at approximately 7 years follow-up, nonmodular constrained condylar knees without stem extensions for primary total knee arthroplasty demonstrate no statistically significant difference in clinical outcomes when compared to standard, posterior-stabilized implants. While the rate of revision was similar between the two cohorts, the modes of failure between the two groups were distinct, as the NMC implants were most commonly revised for femoral component loosening, and the PS implants were all revised for instability.

This study has several limitations that must be noted. First, it is a retrospective review of the results of a single surgeon, and thus, the applicability of these results may be limited. However, the success of NMC prostheses without stem extensions has been similarly reported by other studies $[1,2,7]$. In addition, while those prior studies compared their clinical results to historical controls, this study demonstrates that the NMC cohort performed similarly to the PS cohort at approximately 7 years follow-up. Another limitation is that no strict selection criteria were used to determine which patients would receive PS or NMC prosthesis. However, preoperative criteria are difficult to apply, as the decision to use NMC prosthesis without stem extensions is largely an intraoperative decision, based on the ability to achieve soft tissue balancing and stability of the knee. Lastly, longer follow-up is required to determine if the rate of aseptic loosening with the use of NMC prosthesis without stem extensions will increase in the future.

In this study, we evaluated 190 knees treated with constrained condylar implants without stem extensions for primary total knee arthroplasty, and the mid-term results are encouraging. There were no statistically significant differences in the Hospital for Special Surgery, Knee Society, and SF-12 scores between the NMC and PS groups at approximately 7 years follow-up, and the failure rate between the two groups was nearly identical (4.2\% NMC vs. $4.3 \%$ PS). Anderson et al. [2] reviewed 192 NMC knees, at a mean follow-up of 47 months, and reported a mean Knee Society score of 89 points, and a failure rate of $2.5 \%$, with two failures due to infection, one for aseptic loosening, one for a supracondylar femur fracture, and one for a tibial post

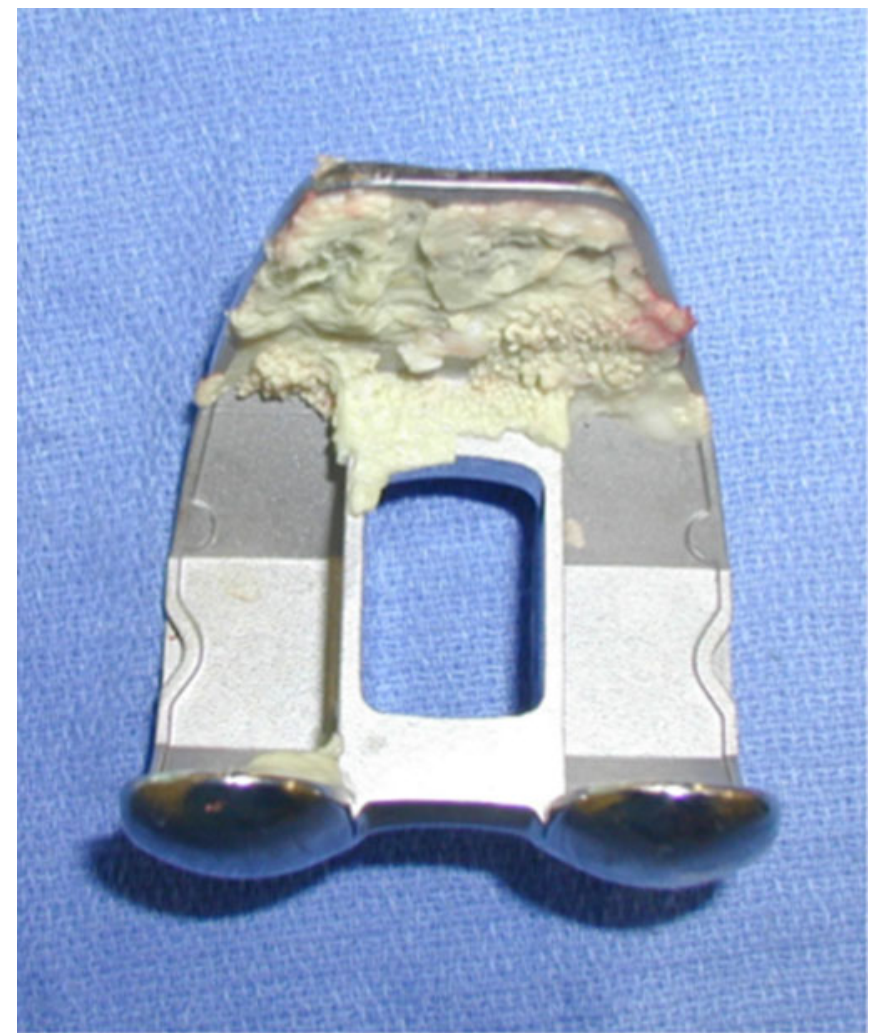

Fig. 7. Image of the posterior aspect of an explanted, NMC femoral component that had been implanted using Palacos cement 
fracture. In our study, at slightly longer follow-up, the mean KSS score was $87.7 \pm 14.5$ in the NMC group, similar to the results achieved in the study by Anderson et al. However, our NMC cohort did have an increased failure rate $(4.2 \%)$, most commonly due to femoral component loosening, and thus, extended follow-up is necessary to determine if aseptic loosening due to increased constraint and stress at the boneimplant interface becomes a more prevalent issue in the future. Of note, femoral component loosening with the use of the NMC implant without stem extensions has previously been reported in a case series of four patients [11]. We hypothesize that only femoral component loosening (and not tibial component loosening) was seen because the tibial component has a small stem that assists with stress transfer and increases stability of that component, while the femoral component does not have any stem attached.

Interestingly, the type of cement used during implantation of the NMC prosthesis seems to have an effect on fixation and mid-term survival of the implant. Twenty-one PS knees were implanted using Palacos cement in our review, none of which demonstrated clinically significant aseptic loosening at approximately 6 years follow-up. However, five out of the nine NMC prostheses implanted using Palacos cement required revision due to femoral component loosening, while aseptic loosening was not appreciated in any of the NMC prostheses implanted with Simplex cement. It is well known that the functional longevity of an implant depends on the fixation achieved at the bone-cement interface, and increased cement intrusion depth improves fixation of the implant. Clinically, based on its handling characteristics, Palacos cement appears more viscous than Simplex cement and requires an increased amount of time to harden. Rey et al., in a bovine cancellous model, assessed the difference in cement intrusion depth between Palacos cement and Simplex cement, at varying levels of injection pressure. They demonstrated that at 20,40 , and $60 \mathrm{lb} / \mathrm{in}^{2}{ }^{2}$ of pressure, Palacos had a cement intrusion depth of 1.4, 2.4, and $2.8 \mathrm{~mm}$, respectively, while at the same pressures, Simplex had an intrusion depth of 2.2, 4.2, and $5.0 \mathrm{~mm}$, respectively [15]. In addition, Jasty et al. [9] demonstrated that the total porosity and mean pore sizes of Palacos cement were inferior to that of Simplex, as Palacos maintained larger pore sizes despite centrifugation, possibly compromising its mechanical strength and decreasing its fatigue life. Therefore, we hypothesize that the decreased intrusion depth of Palacos and its susceptibility to fatigue, combined with the increased stress present at the bone-implant interface with implantation of a NMC prosthesis versus a standard, PS prosthesis, makes the NMC prosthesis susceptible to early, aseptic loosening. During revision surgery of the NMC prostheses implanted with Palacos cement, there was often debonding of the cement from the bone, with cement remaining fixed to the femoral component (Fig. 7). Based on this study, the senior author now only implants NMC prostheses without stem extensions using Simplex bone cement.

While the NMC prostheses in our study were most commonly revised due to femoral component aseptic loos- ening, all six PS prostheses were revised due to persistent knee instability. Instability after both posterior cruciate-substituting and posterior cruciate-retaining total knee arthroplasties is a well-documented complication [14, 16, 18]. While the NMC prostheses without stem extensions in our study demonstrated excellent clinical results, a conventional, posterior-stabilized total knee arthroplasty remains the prosthesis of first choice, as it has an excellent long-term experience, and provides less constraint. However, instability after implantation of PS prosthesis is a concern, and thus, NMC prosthesis without stem extensions is a promising alternative when concerns of instability remain, especially in the older, lower demand patient.

While this study demonstrates that NMC prostheses without stem extensions have excellent clinical results at mid-term follow-up, it must be stressed that appropriate ligamentous balancing and attempting to use the least amount of constraint possible remain important tenets of total knee arthroplasty. Furthermore, the use of a prosthesis with greater constraint increases the loads seen at the boneimplant interface. Therefore, in patients with significant osteopenia or poor bone quality, a constrained condylar system with the use of stem extensions should be considered. In conclusion, this study demonstrates that NMC prostheses without stem extensions can achieve the same clinical results at mid-term follow-up as conventional, PS prostheses. In addition, the authors caution the use of Palacos cement when implanting NMC prosthesis, as this may be associated with early aseptic loosening of the femoral component. However, it must be stressed.

Disclosures Each author certifies that he or she has no commercial associations (e.g., consultancies, stock ownership, equity interest, patent/licensing arrangements, etc.) that might pose a significant conflict of interest in connection with the submitted article.

Each author certifies that his or her institution approved the human protocol for this investigation, that all investigations were conducted in conformity with ethical principles of research, and that informed consent for participation in the study was obtained.

\section{References}

1. Anderson JA, Baldini A, MacDonald JH, Pellicci PM, Sculco TP. Primary constrained condylar knee arthroplasty without stem extensions for the valgus knee. Clin Orthop Relat Res. 2006;442:199-203.

2. Anderson JA, Baldini A, MacDonald JH, Tomek I, Pellicci PM, Sculco TP. Constrained condylar knee without stem extensions for difficult primary total knee arthroplasty. J Knee Surg. 2007;3:195198.

3. Barrack RL, Rorabeck C, Burt M, Sawhney J. Pain at the end of the stem after revision total knee arthroplasty. Clin Orthop Relat Res. 1999;367:216-225.

4. Barrack RL, Stanley T, Burt M, Hopkins S. The effect of stem design on end-of-stem pain in revision total knee arthroplasty. $J$ Arthroplasty. 2004;7 Suppl 2:119-124.

5. Easley ME, Insall JN, Scuderi GR, Bullek DD. Primary constrained condylar knee arthroplasty for the arthritic valgus knee. Clin Orthop Relat Res. 2000;380:58-64.

6. Fehring TK, Odum S, Griffin WL, Mason JB, Nadaud M. Early failures in total knee arthroplasty. Clin Orthop Relat Res. 2001;392:315-318. 
7. Haas SB, Insall JN, Montgomery W,3rd, Windsor RE. Revision total knee arthroplasty with use of modular components with stems inserted without cement. J Bone Joint Surg Am. 1995;11:17001707.

8. Insall JN, Dorr LD, Scott RD, Scott WN. Rationale of the knee society clinical rating system. Clin Orthop Relat Res. 1989;248:13-14.

9. Jasty M, Davies JP, O'Connor DO, Burke DW, Harrigan TP, Harris WH. Porosity of various preparations of acrylic bone cements. Clin Orthop Relat Res. 1990;259:122-129.

10. Lachiewicz PF, Falatyn SP. Clinical and radiographic results of the total condylar III and constrained condylar total knee arthroplasty. J Arthroplasty. 1996;8:916-922.

11. Macdessi SJ, Rich DS, Buly RL. Early femoral component loosening of constrained condylar primary total knee arthroplasties inserted without stems. Journal of Orthopaedics. 2008:20.

12. Merchant AC, Mercer RL, Jacobsen RH, Cool CR. Roentgenographic analysis of patellofemoral congruence. J Bone Joint Surg Am. 1974;7:1391-1396.

13. Nazarian DG, Mehta S, Booth RE,Jr. A comparison of stemmed and unstemmed components in revision knee arthroplasty. Clin Orthop Relat Res. 2002;404:256-262.
14. Pagnano MW, Hanssen AD, Lewallen DG, Stuart MJ. Flexion instability after primary posterior cruciate retaining total knee arthroplasty. Clin Orthop Relat Res. 1998;356:39-46.

15. Rey RM,Jr, Paiement GD, McGann WM, Jasty M, Harrigan TP, Burke DW, Harris WH. A study of intrusion characteristics of low viscosity cement simplex-P and palacos cements in a bovine cancellous bone model. Clin Orthop Relat Res. 1987;215:272-278.

16. Schwab JH, Haidukewych GJ, Hanssen AD, Jacofsky DJ, Pagnano MW. Flexion instability without dislocation after posterior stabilized total knees. Clin Orthop Relat Res. 2005;440:96-100.

17. Sculco TP. The role of constraint in total knee arthoplasty. $J$ Arthroplasty. 2006;4 Suppl 1:54-56.

18. Seon JK, Park SJ, Yoon TR, Lee KB, Moon ES, Song EK. The effect of anteroposterior laxity on the range of movement and knee function following a cruciate-retaining total knee replacement. $J$ Bone Joint Surg Br. 2010;8:1090-1095.

19. Whiteside LA. Correction of ligament and bone defects in total arthroplasty of the severely valgus knee. Clin Orthop Relat Res. 1993;288:234-245.

20. Windsor RE, Insall JN, Wickiewicz TL, Warren RF. The hospital for special surgery knee ligament rating form. Am J Knee Surg. 1988:140-145. 